\title{
Prevalence of HIV-Infection among Sudanese Dental Patients: Using Two Saliva-Testing Assays
}

\section{Nasir EF*}

Preventive Department, King Faisal University, Saudi Arabia

*Corresponding author: Elwalid Fadul Nasir, Associate Professor, Preventive Department, King Faisal University, Saudi Arabia, Email: enasir@kfu.edu.sa

\section{Research Article \\ Volume 5 Issue 2}

Received Date: April 05, 2021

Published Date: May 04, 2021

DOI: $10.23880 /$ eij-16000189

\section{Abstract}

Introduction: till date, HIV infection is still a major health concern. Knowledge of someone's serologic status is important and required for the appropriate provision of services and intervention, and expansion of rapid testing will reduce risk-behaviors and facilitate the prophylaxis of the opportunistic infections. There is a distinguished role of dentistry to lead this issue of optout testing.

Objective: This study was to assess the prevalence of HIV-infection among a group of Sudanese dental patients in using two different assays for saliva (Bionor and OraQuick advance).

Methodology: A cross-sectional hospital-based study was conducted in two phases. First 977 patients were recruited from the outpatients in two dental teaching hospitals (KDTH and UST) in Khartoum state (females $57.6 \%$, mean age 31, SD \pm 8.7 years) using Bionor test. The second phase was implemented in May 2009 including 150 patients (50.7\% males, mean age $34.4, \mathrm{SD} \pm 11.7$ years) recruited from the UST using OraQuick assay.

Results: This is the first study to assess the prevalence of HIV-infection among dental patients in Sudan. Saliva samples with Bionor were all negative, yielding a prevalence of $0 \%$. The second phase three samples were reactive, yielding a prevalence of $2 \%$.

Conclusion: Rapid testing has its multiple advantages that are to be considered and advocated for. The dentistry role in promoting rapid testing and patients screening protocol is highly recommended for infectious diseases and epidemics. OraQuick is a valuable and practical procedure to be used in patients screening in opt-out setting. The results obtained may reflect the real situation of HIV-infection among the study-population.

Keywords: HIV; Saliva; Rapid-Testing; Dental; Sudan

Abbreviations: HIV: Human Immunodeficiency Virus; EIA: Enzyme Immunoassay; VCT: Voluntary Counseling and Testing; SNAP: Sudan National AIDS Program; KDTH: Khartoum Dental Teaching Hospital; UST: University of Science and Technology; FDA: Food and Drug Administration.

\section{Introduction}

Till date, HIV infection is still a major health concern [1]. Approximately, 36.9 million people are living globally with this infection, a marked decrease in the number of 


\section{Epidemiology International Journal}

new infections and AIDS related deaths by $27 \%$ and $56 \%$, respectively, from the period of 2010-17 [2]. The risk of HIV transmission via oral secretion is an important concern issue to dental health professionals [3]. Knowledge of someone's serologic status is important and required for the appropriate provision of services and interventions [4]. Human Immunodeficiency Virus beside blood is found in semen, vaginal fluid, and saliva [5]. HIV-specific antibodies readily found in human saliva provide another potential painless alternative to a blood draw and reliable method of detecting the virus. "Saliva can be used as alternative to blood for detection of HIV antibodies as saliva collection is painless, non-invasive, inexpensive, simple, and rapid" [6].

Expansion of rapid testing will reduce risk-behaviors and facilitate the prophylaxis of the opportunistic infections even in the poorest countries. Where increased testing has been done there is evidence that unsafe sexual practices have been reduced by those with HIV. Another benefit of early detection of HIV is the identification of candidates for experimental therapies [7]. Four findings mandate the increased use of rapid HIV antibody diagnostics both in developing and developed countries for the benefit of public health [8].

The need for immediate HIV test results to make treatment decisions and to assist with prevention strategies portends their increased use in developed countries as well [9]. Having a prospective of future home, on-site, and PointOf-Care (POC), saliva is considered as an excellent alternative to blood, as oral fluid is regarded as a mirror to blood [10]. Saliva sample collection is simple, quick, and non-traumatic and it can be done at low cost. Moreover saliva specimens can be collected without significant infection risks for both patients and operators [11]. The prototype OraScreen dipstick test shows potential for saliva testing in situations where it would be inconvenient to use blood. Further evaluation of saliva testing is warranted on a large sample population with a low prevalence of HIV infection [12]. Blood is generally used for detection of antibodies associated with infectious diseases. However, removal of blood samples can be problematic and is painful for the patient. It requires suitable equipment and skilled staff, both of which may be expensive. Some patients refuse to allow removal of blood samples because they find it painful and traumatic. Removal of blood samples from children, newborns, immunocompromised or overweight subjects is often particularly difficult. In addition, some religions forbid the taking of blood samples.

Thus, it is necessary to develop alternative, simple, painless methods of sampling body fluids that give results as accurate as those obtained with blood samples. Saliva has been suggested as a possible alternative. Epidemiological studies and other reports have shown that saliva may be of value for the detection of HIV antibodies [12]. In situations where blood is difficult or inconvenient to obtain, a substitute to venipuncture offers an attractive alternative [12]. Diagnostic tests for human immunodeficiency virus (HIV) infection have undergone considerable evolution since the first enzyme immunoassay (EIA) and Western blot were introduced 2 decades ago. Newer methods detect infection sooner and yield results much faster. Rapid tests represent a major advance for HIV screening [13]. Rapid HIV tests are widely used in resource-poor settings, especially in developing countries. ELISA and Western blot were not feasible for small laboratories in many developing countries where resources are limited and electricity may not be consistently available. It was considered that a non-invasive saliva screening EIA could be an advantageous alternative [14].

Saliva collection is easy to perform, non-invasive, safe to use and thus provides alternative method to blood collection [15]. Has the advantages of being simple and non-invasive. This paper reviews the field and discusses antibody testing of dental patients [16]. Rapid HIV tests offer additional advantages of low cost and same-day results and are likely to gain increasing acceptance for HIV screening and diagnosis in both developed and developing countries [9]. Screening with combinations of rapid HIV tests proved to be less expensive than the ELISA/Western blot algorithm [7]. Rapid HIV testing using oral specimens in the RIDOC jail was feasible and preferred by correctional staff [17]. The majority of subjects supported routine HIV testing in jail and the concept of partner notification services. In this population of jail detainees, rapid HIV testing was feasible and highly acceptable [18].

The presence of HIV-antibodies in the saliva makes saliva sample a promising means of testing for HIV antibodies. Salivary antibody testing for HIV is proving to be a sensitive and specific procedure, especially useful for epidemiological studies. HIV antibodies are found in human saliva and can be detected by sensitive ELISA. Testing saliva is effective for determining HIV status early in seroconversion. Saliva has been recommended as an alternative non-invasive specimen for detection of antibodies to human immunodeficiency virus (HIV) because of the inherent disadvantages of using serum for such testing. The sensitivity of saliva for HIV antibody detection using the modified test protocol was found to be $95 \%$ by GENELAVIA MIXT ELISA and $97 \%$ by DETECT-HIV ELISA, while the specificity for both was $100 \%$. Saliva is a safe and cost-effective alternative to serum for HIV antibody detection for most surveillance purposes but not for diagnostic purposes [19].

The IgG concentration in oral mucosal transudates, however, is less than that in plasma but higher than that in whole saliva, the level of IgG in oral fluid is about $1 / 800$ that 


\section{Epidemiology International Journal}

of serum [20]. Best results are obtained with oral fluids that are rich in IgG, since the primary humoral immune response to HIV infection involves mainly this class of antibodies. In studies with saliva conducted between 1986 and 1991, the concordance between positive serum tests and positive saliva tests for the detection of HIV antibodies ranged from 70 to $100 \%$. Rapid HIV tests demonstrate sensitivities and specificities comparable to those of enzyme-linked immunoassays (ELISAs) currently used for screening.

\section{Objectives}

With the realization of the importance of rapid testing and its impact on prevention, service provision as well as early access to proper treatment, the distinguished role of dentistry to lead this issue of opt-out testing, this study aimed at assessing the prevalence of HIV-infection among dental patients in Sudan using both Bionor saliva test and OraQuick advance assay.

\section{Materials and Methods}

The Republic of the Sudan is a country in Sub-Saharan Africa. The country has religious, ethnic and linguistic diversity with up to 400 spoken languages [21]. The population of Sudan is about 39.379.358 estimation [22]. Sentinel surveillance was conducted on a limited scale in 2004 and 2005 by Sudan National AIDS Program (SNAP), reported prevalence rates of sex workers $4.4 \%$ and men-whohave sex with men 9\%; as well as among clients attending the Voluntary Counseling and Testing (VCT) services to be $5-15 \%$. The total number of people living with HIV in the country is about 522,720 with a prevalence among adults of reproductive age $15-49$ years is about $2.6 \%$ [23]. Long-term war, the economic crisis, urbanization, and borders with many countries some of which have high HIV prevalence, provide an enabling environment for high risk sexual behaviors and rapid spread of HIV infection. One objective of the Sudan HIV/AIDS national strategic plan is to maintain the level of HIV prevalence at less than $2 \%$ by 2009 [23].

\section{Study Design and Participants}

This cross-sectional hospital-based prevalence study was carried out in two phases: first phase was from MarchJuly 2008 using Bionor assays, Survey participants were recruited from outpatients at two dental teaching hospitals in Khartoum state, Khartoum Dental Teaching Hospital (KDTH) and University of Science and Technology (UST). A sample size of 1200 patients was assumed to be satisfactory for a two-sided test assuming the proportion of dental care utilization in the previous 2 years to be 0.15 and 0.20 in patients with respectively low- and high education, a significance level of $5 \%$ and a power of $80 \%$. The second phase was from 4th to 28th of May 2009 using OraQuick assay. A total of 150 kits were provided for OraQuick test. Patients were recruited from UST dental outpatient clinic. All patients attending during the study period were invited to participate in the study. In the two phases, the inclusion criteria were; all patients who are over 18 years of age, with reported unknown HIV/AIDS status, coming with a dental complain. Reason for not participating was mainly due to time limitation on the part of patients and harried intention to receive dental treatment.

\section{Data Collection: Saliva Samples Collection for Bionor Test (MucoSafe TM)}

The Bionor ${ }^{\mathrm{TM}}$ test captures IgG-antibodies against HIV-1 $\& 2$ and their subgroups using a Magnetic Particle Enzyme Immuno Assay (EIA) method. The tests are very easy to use and results can be obtained in less than 2 hours [24]. Reading of results is simple and clear-cut, and performed visually. Oral fluids samples were collected by the researcher, who was trained by the manufacturer prior to the conduction of the study, using MucoSafe TM (Norway) saliva collection devices according to the manufacturer's instructions. The MucoSafe TM collection strip was placed in the mouth between the lower gums and buccal mucosa rubbed and left in the cavity for 3 to 5 minutes to absorb oral fluids until the absorbent membrane is saturated. It was then placed in the special bag for transportation and sent to the lab for further processing. They were kept in the refrigerator until processed. All samples were processed at the UST laboratory at the faculty of the laboratory sciences. Three qualified technicians had been assigned to process the samples under supervision and control of the senior virologist. The laboratory process followed the manufacturer's manual of instructions, and results were interpreted according to the manufacturer's protocol [25].

\section{Saliva Samples for OraQuick}

The OraQuickR advance TM Rapid HIV-1/2 antibody test is a single use qualitative immunoassay to detect antibodies to HIV-1 and 2 in oral fluid, finger stick whole blood, venipuncture whole blood, and plasma specimens. The specimen is collected by swabbing the upper and lower gums with the test device. The patient places a pad against his or her teeth in supragingival position, and oral transudate is collected in a manner similar to capillary action [26]. The specimen and test device are added to a vial of developer solution, and test results are read 20-40 minutes later. All participants had been informed about the objectives, procedures and benefits of the study prior to the participation in the study. Participants had been asked to sign a (twopart) written consent form. The first part was accepting to be included in the study and the second part to receive 


\section{Epidemiology International Journal}

information regarding the results of the HIV-test. Participants who signed for knowing their status had provided their telephone numbers for further communication. Pre-test and post-test counseling was arranged before the conduction of the study. Arrangements for referral of patients with positive results to the HIV-special clinics for further management had been arranged with the respective clinics. All samples for Bionor $^{\mathrm{TM}}$ test were coded prior to sending to the laboratory to secure confidentiality. Results were sent back with the codes and then decoded by the researcher and related to the patients' profiles.

\section{Results and Discussion}

This is the first study to attempt to determine the prevalence of HIV-infection among dental outpatients in Sudan, and also one of the rare studies to explore HIV- infection in dental clinics [27].

\section{Sample profile}

A total of 977 patients' saliva samples (mean age 31 and SD 8.7) have been processed using Bionor test. From UST were 491 patients (50.3\%) and 486 patients (49.7\%) were from KDTH. Table 1 gives the percentage distribution of participants' socio-demographic characteristics (predisposing factors) according to hospital of attendance. The patients attending UST were less frequently males (35.2\% versus $49.6 \%)$, less frequently in the younger age group ( $47.6 \%$ and $59.1 \%$ ), and had more frequently travelled outside Sudan (41.5\% versus $27.6 \%$ ) as compared to their KDTH counterparts (Table 1). All patients in this group had negative results, which makes the prevalence among dental patients to be $0.0 \%$.

\begin{tabular}{|c|c|c|c|}
\hline Characteristic & UST \% (n) & KDTH \% (n) & Total \% (n) \\
\hline \multicolumn{4}{|c|}{ Gender } \\
\hline Male & $35.2(173)$ & $49.6(241)$ & $42.4(414)$ \\
\hline Female & $64.8(318)^{* *}$ & $50.4(245)$ & $57.6(563)$ \\
\hline \multicolumn{4}{|c|}{ Age } \\
\hline$\leq 29$ & $47.6(233)$ & $59.1(287)$ & $53.3(520)$ \\
\hline$\geq 30$ & $52.4(257)^{* *}$ & 40.9 (199) & $46.7(456)$ \\
\hline \multicolumn{4}{|c|}{ Marital status } \\
\hline Single & $43.0(211)$ & $47.5(231)$ & $45.2(442)$ \\
\hline In relationship & $57.0(280)$ & $52.5(255)$ & $54.8(535)$ \\
\hline \multicolumn{4}{|c|}{ Education } \\
\hline Primary/secondary & $48.3(237)$ & $46.1(224)$ & $47.2(461)$ \\
\hline University and higher & $51.7(254)$ & $53.9(262)$ & $52.8(516)$ \\
\hline \multicolumn{4}{|c|}{ Profession } \\
\hline Unemployed, other & $62.0(304)$ & $58.8(286)$ & $60.5(590)$ \\
\hline Technical, office, skilled labor & $38.0(186)$ & $41.2(200)$ & $39.5(386)$ \\
\hline \multicolumn{4}{|c|}{ Travelling inside Sudan } \\
\hline Yes & $85.9(420)$ & $84.2(409)$ & $85.0(829)$ \\
\hline No & $14.1(69)$ & $15.8(77)$ & $15.0(146)$ \\
\hline \multicolumn{4}{|c|}{ Travelling outside Sudan } \\
\hline Yes & $41.5(203)^{* *}$ & $27.6(134)$ & $34.6(337)$ \\
\hline No & $58.5(286)$ & $72.4(352)$ & $65.4(638)$ \\
\hline
\end{tabular}

Table 1: Frequency and percentage distribution of dental patients' socio-demographic characteristics (predisposing factors) in UST and KDTH hospitals.

\section{OraQuick Sample Profile}

A total of 150 patients were tested using OraQuick. Seventy four (49.3\%) were females; with a total mean age of
34.35 years and a SD of 11.76 years. Among these patients, three had reactive tests. They were as follows; 21-yearold male, 50-year-old male, and 53-year-old female. This makes the prevalence to be $2 \%$. In a recent study in India 


\section{Epidemiology International Journal}

using saliva and serum samples, ELISA results were $99 \%$ sensitive and $100 \%$ specific for HIV antibodies-there was 1 false positive-while for the serum samples, they were $100 \%$ for both measures. "Saliva can be used as alternative to blood for detection of HIV antibodies as saliva collection is painless, non-invasive, inexpensive, simple, and rapid," Like all rapid tests oral-fluid-based tests positive results must be considered as primary and should be confirmed with blood or plasma-based approved tests.

Despite reported high accuracy of oral-based tests, their accuracy and clinical utility also may depend on disease prevalence, clinical presentation and type of study populations [28]. The results obtained may reflect the real situation of HIV-infection among the study-population, while some possible factors (limitations) might have contributed to suspected false negatives; the first is the low prevalence of HIV-infection in Sudan as there is reported practical implication with lower specificity especially in population with low HIV prevalence [29]. There is as well the possibility of self-selection bias, as it is known that very sick persons tend not to attend dental clinics in seeking their prioritized medical problems [30].

Persons with HIV-related risk-behavior may prefer not to participate in such studies to avoiding stigma and discrimination in treatment as well as management required. Human-errors related to different procedure's steps (sample collection, storing and laboratory process) may have also occurred. As it has been shown the processing of the saliva samples is less critical (with certain limits) in testing for seropositivity [31]. The small sample size on the second phase of the study is to be considered as a limitation in generalizing these results.

The most likely factor is the ability of the collection device to collect an amount of oral fluid very rich with IgG, as this is known as the most important condition required in oral fluid. Best results are obtained with oral fluids that are rich in IgG [32]. Among the collection devices studied, Orasure device resulted in the highest sensitivity and specificity scores for HIV-antibodies detection. Many studies have been carried out to assess the accuracy of OraQuick. In Namibia a study claimed as the first field evaluation of OraQuick and OraSure devices in a resource-limited setting in Southern Africa, has proved that OraSure has the specificity of $99.5 \%$ and sensitivity of $97.1 \%$ [33], OraQuick also demonstrated its utility for detecting HIV subtypes [34]. Orasure device has been found to be able to collect the highest concentration of IgG among other several devices [35]. So many studies have shown the validity of OraSure as well as its suitability as surveying tool in prevalence studies among general population [36]. OraSure being easy to use with no laboratory work needed, shorter time to give the results, and very high acceptance by health providers and patients as well. The United States Food and Drug Administration (FDA) have approved OraQuick since 2002. Very too little literature on the validity of Bionor was available. There was no accessible data on the accuracy and validity of Bionor assay. Any new diagnostic test to get acceptance, an extensive data of publication supporting the science should be available [37].

\section{Conclusion}

We could conclude that oral fluid is an appealing medium for HIV-testing. Rapid testing has its multiple advantages that are to be considered and advocated for. The dentistry role in promoting rapid testing and patients screening protocol is highly recommended. HIV-infection and oral health are interrelated. OraQuick is a valuable and practical procedure to be used in patients screening in opt-out setting. The accuracy of OraQuick puts this assay as a priority method in carrying such services and studies. Due to different limitations we have mentioned, it seems that the results of both surveys are not conclusive. More studies are to be carried out to precisely determine the burden of HIV-infection in dental as well as other outpatients setting in Sudan.

\section{References}

1. Shaw ML (2020) Saliva May Be a Reliable Alternative to Blood for HIV Antibody Testing. AJMC.

2. (2018) UNAIDS.

3. Campo J, Perea MA, del Romero J, Cano J, Hernando V, et al. (2006) Oral transmission of HIV, reality or fiction? An update. Oral Dis 12(3): 219-228.

4. De Cock KM, Bunnell R, Mermin J (2006) Unfinished business-expanding HIV testing in developing countries. N Engl J Med 354(5): 440-442.

5. Chinnapolamada JR, Chiranjeevi SA, Tupalli AR, Erugula SR (2015) Sialodiagnosing' HIV infection: A dissected review. Journal of Indian Academy of Oral Medicine and Radiology 27(2): 213-218.

6. Vohra P, Belkhode V, Nimonkar S, Potdar S, Bhanot R, et al. (2020) Evaluation and diagnostic usefulness of saliva for detection of HIV antibodies: A cross-sectional study. J Family Med Prim Care 9(5): 2437-2441.

7. Rhame FS, Maki DG (1989) The case for wider use of testing for HIV infection. N Engl J Med 320(19): 12481254 .

8. Spielberg F, Kassler WJ (1996) Rapid testing for HIV antibody: a technology whose time has come. Ann Intern Med 125(6): 509-511. 


\section{Epidemiology International Journal}

9. Branson BM (2000) Rapid Tests for HIV Antibody. AIDS Reviews 2(2): 76-83.

10. Paul LA, Malamud D (2008) Point-Of-Care Diagnosis Salivary Diagnostics. In: DT Wong (Eds.), WellyBlackwell, pp: 136-149.

11. Polimeni A, Gallottini L, Resi JP (1995) Salivary HIV antibodies. Review of current data and future prospectives. Minerva Stomatol 44(12): 583-590.

12. Sangare KA, Koffi AR, Coulibaly IM, Doulourou C (1997) Could saliva be used to detect HIV seroconversion? Sante 7(5): 303-307.

13. Branson BM (2007) State of the art for diagnosis of HIV infection. Clin Infect Dis 45(4): 221-225.

14. King SD, Wynter SH, Bain BC, Brown WA, Johnston JN, et al. (2000) Comparison of testing saliva and serum for detection of antibody to human immunodeficiency virus in Jamaica, West Indies. J Clin Virol 19(3): 157-161.

15. Anil S, Beena VT, Nair RG, Varghese BJ (1995) Detection of HIV antibodies in saliva and its implications. Indian J Dent Res 6(3): 95-98.

16. Scully C (1997) HIV topic update: salivary testing for antibodies. Oral Dis 3(4): 212-215.

17. Beckwith CG, Bazerman L, Cornwall AH, Patry E, Poshkus $M$, et al. (2011) An evaluation of a routine opt-out rapid HIV testing program in a Rhode Island jail. AIDS Educ Prev 23(3): 96-109.

18. Desai AA, Latta ET, Spaulding A, Rich JD, Flanigan TP (2002) The importance of routine HIV testing in the incarcerated population: the Rhode Island experience. AIDS Educ Prev 14(5): 45-52.

19. Kamat HA, Adhia M, Koppikar GV, Parekh BK (1999) Detection of antibodies to HIV in saliva. Natl Med J India 12(4): 159-161.

20. Malamud D (1997) Oral diagnostic testing for detecting human immunodeficiency virus-1 antibodies: a technology whose time has come. Am J Med 102(4): 9-14.

21. Ministry of Health Sudan and N. A. Control (2008) HIV/ AIDS in Northen Sudan.

22. WHO (2008) Epidemiological Fact Sheet on HIV and AIDS.

23. UNGA (2008) United Nations General Assembly Special Session on HIV/AIDS (UNGASS).
24. Sommerfelt MA, Ohlsson I, Flolid I, Thorstensson R, Sorensen B (2004) A simple semi-rapid HIV-1\&2 confirmatory immunoassay using magnetic particles. J Virol Methods 115(2): 191-198.

25. (2009) Bionor.

26. Vernillo AT, Calpan AL (2007) Routine HIV testing in dental practice: can we cross the Rubicon?. J Dent Educ 71(12): 1534-1539.

27. de Almeida OP, de Souza Filho FJ, Scully C, Line SR, Porter S (1997) HIV prevalence in dental outpatients in Brazil. Oral Surg Oral Med Oral Pathol Oral Radiol Endod 84(4): 365-367.

28. Pai NP (2007) Rapid oral fluid-based point-of-care HIV testing: applicability in developing countries. Indian J Med Res 126(3): 171-173.

29. Delaney KP, Branson BM, Uniyal A, Kerndt PR, Keenan PA, et al. (2006) Performance of an oral fluid rapid HIV$1 / 2$ test: experience from four CDC studies. AIDS 20(12): 1655-1660.

30. Dobalian A, Andersen RM, Stein JA, Hays RD, Cunningham WE, et al. (2003) The impact of HIV on oral health and subsequent use of dental services. J Public Health Dent 63(2): 78-85.

31. Enno CI, Veerman AV (2008) Processing and storage of saliva samples. Salivary Diagnostics. In: Wong DT (Eds.), Wlley-Blackwell, pp: 69-76.

32. Hodinka RL, Nagashunmugam T, Malamud D (1998) Detection of human immunodeficiency virus antibodies in oral fluids. Clin Diagn Lab Immunol 5(4): 419-426.

33. Hamers RL, de Beer IH, Kaura H, van Vugt M, Caparos L, et al. (2008) Diagnostic accuracy of 2 oral fluid-based tests for HIV surveillance in Namibia. J Acquir Immune Defic Syndr 48(1): 116-118.

34. Holguin A, Gutierrez M, Portocarrero N, Rivas P, Baquero M (2009) Performance of OraQuick Advance Rapid HIV$1 / 2$ Antibody Test for detection of antibodies in oral fluid and serum/plasma in HIV-1+ subjects carrying different HIV-1 subtypes and recombinant variants. J Clin Virol 45(2): 150-152.

35. Malamud D (1997) Oral Diagnostic Testing for Detecting Human Immunodeficiency Virus-1 Antibodies: A Technology Whose Time Has Come. Am J Med 102 (4): 9-14.

36. Pascoe SJ, Langhaug LF, Mudzori J, Burke E, Hayes R, et al. (2009) Field evaluation of diagnostic accuracy of an 


\section{Epidemiology International Journal}

oral fluid rapid test for HIV, tested at point-of-service sites in rural Zimbabwe. AIDS Patient Care STDS 23(7): 571-576.
37. Smith SR, Wong DT (2008) Saliva diagnostics-a new industry. Salivary Diagnostics. In: Wong DT (Eds.), WellyBlackwell, pp: 288-294. 